\title{
THE DOMAIN OF DEPENDENCE INEQUALITY FOR SYMMETRIC HYPERBOLIC SYSTEMS ${ }^{1}$
}

\author{
BY CALVIN H. WILCOX \\ Communicated by Ralph Phillips, August 19, 1963
}

1. Introduction. Energy propagates through space with finite speed. This is a characteristic feature of wave propagation phenomena and, indeed, it is a postulate in relativistic physics. Recently $[3 ; 4]$ the author demonstrated this property by means of $a$ priori "domain of dependence" inequalities for solutions of initial-boundary value problems for Maxwell's equations and second order hyperbolic equations and used the inequalities to prove existence, uniqueness and regularity theorems for these problems. In this announcement the results are extended to the most general systems of linear partial differential equations that admit an energy integral, the symmetric hyperbolic systems of K. O. Friedrichs [1].

The systems have the form

(1.1) $E_{\alpha \beta}(x) \frac{\partial u_{\beta}}{\partial t}=A_{\alpha \beta}^{i}(x) \frac{\partial u_{\beta}}{\partial x_{i}}+B_{\alpha \beta}(x) u_{\beta}+f_{\alpha}(x, t)(\alpha=1,2, \cdots, m)$, where $E_{\alpha \beta}, A_{\alpha \beta}^{i}$ and $B_{\alpha \beta}$ are real-valued functions of $x=\left(x_{1}, x_{2}, \cdots, x_{n}\right)$ $\in R^{n}$ and $u_{\beta}=u_{\beta}(x, t)$ and $f_{\alpha}$ are real-valued functions of $x$ and $t \in R^{1}$. The summation convention is used; i.e., repeated indices $i$ and $\beta$ are summed over their ranges $(1 \leqq i \leqq n, 1 \leqq \beta \leqq m)$. System (1.1) may also be written in matrix notation

$$
E \frac{\partial u}{\partial t}=A^{i} \frac{\partial u}{\partial x_{i}}+B u+f
$$

where $E=\left(E_{\alpha \beta}\right), A^{i}=\left(A_{\alpha \beta}^{i}\right)$ and $B=\left(B_{\alpha \beta}\right)$ are $m \times m$ matrices and $u=\left(u_{\alpha}\right)$ and $f=\left(f_{\alpha}\right)$ are $m \times 1$ (column) matrices.

A system (1.1) is symmetric hyperbolic if $E$ and $A^{i}(i=1,2, \cdots, n)$ are symmetric and $E$ is positive definite. The form $\eta=\frac{1}{2} E_{\alpha \beta} u_{\alpha} u_{\beta}$ is interpreted as an "energy density" (energy per unit volume). The forms $\Sigma_{i}=-\frac{1}{2} A_{\alpha \beta}^{i} u_{\alpha} u_{\beta}$ are interpreted as the components of a "Poynting vector" describing the flow of power (energy per unit area per unit time). They are related by the "conservation of energy law"

$$
\frac{\partial \eta}{\partial t}+\frac{\partial \Sigma_{i}}{\partial x_{i}}=\frac{1}{2}\left(B_{\alpha \beta}+B_{\beta \alpha}-\frac{\partial A_{\alpha \beta}^{i}}{\partial x_{i}}\right) u_{\alpha} u_{\beta}+f_{\alpha} u_{\alpha} .
$$

${ }^{1}$ Sponsored by the Mathematics Research Center, United States Army, Madison, Wisconsin, under Contract No. DA-11-022-ORD-2059. 
The energy per unit time entering a domain $\Omega \subset R^{n}$ through its boundary $\partial \Omega$ is

$$
\int_{\partial \Omega} \Sigma_{i} n_{i} d S=\int_{\partial \Omega} \eta v_{i} n_{i} d S \quad\left(n_{i}=\text { normal vector on } \partial \Omega\right)
$$

where $v_{i}=\Sigma_{i} / \eta$. Thus, $v_{i}$ may be interpreted as a "velocity" of energy flow, which suggests that the speed with which energy propagates in $\Omega$ is bounded above by

$$
c=\sup \frac{-A_{\alpha \beta}^{i}(x) \xi_{\alpha} \xi_{\beta} n_{i}}{E_{\alpha \beta}(x) \xi_{\alpha} \xi_{\beta}},
$$

where the supremum is over $x \in \Omega, \xi \in R^{m}$ and unit vectors $n_{i}\left(n_{i} n_{i}=1\right)$. This property of $c$ is verified below ( $\$ 3$ ) by the domain of dependence inequality.

This announcement deals with initial-boundary value problems for (1.1) in domains $\Omega$, subject to dissipative, local boundary conditions on $\partial \Omega$. Precise definitions of the boundary conditions are given in $\$ 2$. The domain of dependence inequality is formulated and proved in $\$ 3$. Its application to existence, uniqueness, and regularity theorems for initial-boundary value problems, together with complete proofs of all the results and their application to particular systems, will be given elsewhere (see [5]).

Throughout this paper, $\Omega$ denotes an arbitrary domain (open connected set) in $R^{n}$, and $E_{\alpha \beta}(x), A_{\alpha \beta}^{i}(x)$ and $B_{\alpha \beta}(x)$ are assumed to be defined (almost everywhere) in $\Omega$ and to satisfy the conditions

$$
E_{\alpha \beta}(x)=E_{\beta \alpha}(x), A_{\alpha \beta}^{i}(x)=A_{\beta \alpha}^{i}(x), B_{\alpha \beta}(x) \text { and } \partial A_{\alpha \beta}^{i}(x) / \partial x_{i}
$$

(which is defined as a distribution) are bounded, Lebesgue-measurable functions in $\Omega$, and

(1.5) There exists a positive constant $E^{0}$ such that

$$
E_{\alpha \beta}(x) \xi_{\alpha} \xi_{\beta} \geqq E^{0} \xi_{\alpha} \xi_{\alpha} \quad \text { for } x \in \Omega \text { and } \xi \in R^{m} .
$$

2. Boundary conditions. If $\phi=\left(\phi_{\alpha}\right) \in C_{0}^{1}(\Omega)$ then

$$
(A \phi)_{\alpha}=A_{\alpha \beta}^{i} \frac{\partial \phi_{\beta}}{\partial x_{i}}+\frac{1}{2} \frac{\partial A_{\alpha \beta}^{i}}{\partial x_{i}} \phi_{\beta} \quad(\alpha=1,2, \cdots, m)
$$

defines an operator $A: C_{0}^{1}(\Omega) \rightarrow L_{2}(\Omega)$, and $A^{+}$, the formal adjoint of $A$, equals $-A$. This suggests the

Definition. If $u$ is in $L_{2}(\Omega)$, then we say that $A u$ exists and is 
equal to $v$ in $L_{2}(\Omega)$ provided that

$$
\int_{\Omega} u_{\alpha}(A \phi)_{\alpha} d x+\int_{\Omega} v_{\alpha} \phi_{\alpha} d x=0 \quad \text { for all } \phi \in C_{0}^{1}(\Omega) \text {. }
$$

The space $L_{2}(A ; \Omega)=\left\{u: u\right.$ and $A u$ are in $\left.L_{2}(\Omega)\right\}$ is a separable Hilbert space with respect to the scalar product

$$
(u, v)_{L_{2}(A ; \Omega)}=\int_{\Omega}\left\{u_{\alpha} v_{\alpha}+(A u)_{\alpha}(A v)_{\alpha}\right\} d x .
$$

In classical theory, the set of functions $u$ which satisfy a linear homogeneous boundary condition on $\partial \Omega$ and have $A u \in L_{2}(\Omega)$ form a linear space containing $C_{0}^{1}(\Omega)$. This suggests the

Definition. A boundary condition $\Gamma$ for $A$ is a closed linear subspace $\Gamma$ of $L_{2}(A ; \Omega)$ such that $C_{0}^{1}(\Omega) \subset \Gamma$.

Each boundary condition $\Gamma$ for $A$ determines an "adjoint" boundary condition $\Gamma^{*}$ for $A$ defined by

$$
\Gamma^{*}=L_{2}(A ; \Omega) \cap\left\{v: \int_{\Omega}\left\{u_{\alpha}(A v)_{\alpha}+(A u)_{\alpha} v_{\alpha}\right\} d x=0 \text { for all } u \in \Gamma\right\} \text {. }
$$

It follows that $\left(\Gamma^{*}\right)^{*}=\Gamma$. Thus, boundary conditions for $A$ occur in adjoint pairs.

Definition. $\Gamma$ is a dissipative boundary condition for $A$ provided that

$$
\int_{\Omega} u_{\alpha}(A u)_{\alpha} d x \leqq 0 \quad \text { for all } u \in \Gamma .
$$

This concept is equivalent to that of a dissipative operator due to R. S. Phillips [2]; see [5].

Definition. $\Gamma$ is a local boundary condition for $A$ provided that $\phi u \in \Gamma$ whenever $\phi \in C_{0}^{1}\left(R^{n}\right)$ and $u \in \Gamma$.

Examples of local, dissipative boundary conditions are provided by the classical systems of acoustics, electromagnetics and elasticity. With each is associated a pair of "natural" boundary conditions, corresponding to hard and soft boundaries in acoustics, perfect electrical and magnetic conductors in electromagnetics, and fixed and free boundaries in elasticity; see [5] where this is verified and a class of systems and boundary conditions generalizing these is described.

3. The domain of dependence inequality. Each boundary condition $\Gamma$ for $A$ is a closed subspace of $L_{2}(A ; \Omega)$. Hence, $\Gamma$ is a separable Hilbert space relative to the scalar product of $L_{2}(A ; \Omega)$. If $I=\{t: 0<t<T\}$ then the space 


$$
F=L_{2}(I ; \Gamma) \cap L_{2}^{1}\left(I ; L_{2}(\Omega)\right)
$$

(see $[4, \S 2]$ for definitions) defines a class of functions $u=u(x, t)$ for which

$$
E \frac{\partial u}{\partial t}, A^{i} \frac{\partial u}{\partial x_{i}}=A u-\frac{1}{2} \frac{\partial A^{i}}{\partial x_{i}} u \text { and } B u
$$

exist in $L_{2}(Q), Q=\Omega \times I$, and $u$ satisfies the boundary condition $\Gamma(u(t) \in \Gamma$ for almost every $t \in I)$. The domain of dependence inequality is concerned with the related space $F^{\text {loo }}$ for which $E(\partial u / \partial t)$, etc., are in $L_{2}^{\text {loc }}(Q)$. To describe it let

$\Gamma^{\mathrm{vox}}=\Gamma \cap\{u: u$ is equivalent to zero outside a bounded set $K \subset \Omega\}$, and

$$
\begin{aligned}
\Gamma^{100}= & L_{2}^{100}(A ; \Omega) \\
& \cap\left\{u: \int_{\Omega}\left\{u_{\alpha}(A v)_{\alpha}+(A u)_{\alpha} v_{\alpha}\right\} d x=0 \text { for all } v \in\left(\Gamma^{*}\right)^{\text {vox }}\right\}
\end{aligned}
$$

and define

$$
\begin{aligned}
F^{\text {loc }}= & \left\{u: u \in L_{2}\left(I ; L_{2}(A ; K \cap \Omega)\right) \cap L_{2}^{1}\left(I ; L_{2}(K \cap \Omega)\right)\right. \text { for each } \\
& \text { bounded measurable set } K \subset R^{n} \text {, and } u(t) \in \Gamma^{\text {loo }} \text { for al- } \\
& \text { most all } t \in I\} .
\end{aligned}
$$

Theorem (Domain of DEPENDENCE INEQUALITy). Let $\Gamma$ be a local, dissipative boundary condition for $A$. Then the speed $c$ defined by (1.3) is finite and every $u \in F^{\text {loo }}$ satisfies

$$
\begin{aligned}
& \int_{\Omega \cap S\left(x^{0}, a\right)} E_{\alpha \beta} u_{\alpha}(T) u_{\beta}(T) d x \\
& \leqq e^{k T}\left[\int_{\Omega \cap S\left(x^{0}, a+c T\right)} E_{\alpha \beta} u_{\alpha}(0) u_{\beta}(0) d x+2 \int_{Q \cap C\left(x^{0}, a\right)} e^{-k t} f_{\alpha} u_{\alpha} d x d t\right]
\end{aligned}
$$

where

$$
\begin{aligned}
f & =E \frac{\partial u}{\partial t}-A u-\left(B-\frac{1}{2} \frac{\partial A^{i}}{\partial x_{i}}\right) u \\
S\left(x^{0}, a\right) & =\left\{x:\left|x-x^{0}\right| \leqq a\right\} \text { and } \\
C\left(x^{0}, a\right) & =\left\{(x, t):\left|x-x^{0}\right| \leqq a+c(T-t), 0 \leqq t \leqq T\right\} .
\end{aligned}
$$

The constant $k$ depends on $n$ and the bounds for the coefficients only. 
Proof. The finiteness of $c$ follows from (1.4) and (1.5). The proof is omitted. Also, only the special case $B=\frac{1}{2} \partial A^{i} / \partial x_{i}$ is treated. To prove (3.1), take $\delta>0$ and let $\psi_{\delta} \in C_{0}^{\infty}\left(R^{1}\right)$ have the properties $\psi_{\delta}(\tau) \equiv 0$ for $\tau \geqq \delta, \psi_{\delta}(\tau) \equiv 1$ for $\tau \leqq-\delta$, and $\psi_{\delta}^{\prime}(\tau) \leqq 0$ everywhere (so $0 \leqq \psi_{\delta}(\tau)$ $\leqq 1$ everywhere). Put $\phi(x, t)=\psi_{\delta}(\tau), \tau=\left|x-x^{0}\right|-a-c(T-t)$. Then $\phi \in C_{0}^{\infty}(\bar{Q})$ for $\delta$ sufficiently small, and $\phi$ tends to the characteristic function of $C\left(x^{0}, a\right)$ when $\delta \rightarrow 0+$.

Let $u \in F^{\text {loc }}$, define $f \equiv E(\partial u / \partial t)-A u$ and consider

$$
\int_{Q} f_{\alpha} u_{\alpha} \phi d x d t=\int_{Q} \phi u_{\alpha} E_{\alpha \beta} \frac{\partial u_{\beta}}{\partial t} d x d t-\int_{Q} \phi u_{\alpha}(A u)_{\alpha} d x d t=I_{1}-I_{2} .
$$

Integration by parts with respect to $t$ gives [4, Theorem 2.3]

$$
\left.I_{1}=\frac{1}{2} \int_{\Omega} \phi E_{\alpha \beta} u_{\alpha} u_{\beta}\right]_{0}^{T} d x-\frac{1}{2} \int_{Q}\left(E_{\alpha \beta} u_{\alpha} u_{\beta}\right) \frac{\partial \phi}{\partial t} d x d t \text {. }
$$

Next, $\sqrt{ }(\phi(\cdot, t)) \in C_{0}^{1}\left(R^{n}\right)$ for each $t \in I$, and $u(\cdot, t) \in \Gamma^{\text {loo }}$ for almost every $t \in I$. Hence, $\sqrt{ }(\phi(\cdot, t)) u(\cdot, t) \in \Gamma$ because $\Gamma$ is a local boundary condition for $A$. Thus, since $\Gamma$ is dissipative for $A$,

$$
0 \geqq \int_{\Omega} \sqrt{ }(\phi(x, t)) u_{\alpha}(x, t)(A \sqrt{ }(\phi(x, t)) u(x, t))_{\alpha} d x
$$

for almost all $t \in I$.

Now, $(A \psi u)_{\alpha}=\psi(A u)_{\alpha}+A_{\alpha \beta}^{i}\left(\partial \psi / \partial x_{i}\right) u_{\beta}$, whence (3.4) may be written

$$
\begin{aligned}
0 \geqq & \int_{\Omega} \phi(x, t) u_{\alpha}(x, t)(A u(x, t))_{\alpha} d x \\
& \left.+\int_{\Omega} \sqrt{ }(\phi(x, t)) \frac{\partial \sqrt{ }(\phi(x, t))}{\partial x_{i}} A_{\alpha \beta}^{i} u_{\alpha}(x, t) u_{\beta}(x, t) d x \quad \text { (almost all } t \in I\right) .
\end{aligned}
$$

Integrating over $t \in I$ and using

gives

$$
\sqrt{ } \phi \frac{\partial \sqrt{ } \phi}{\partial x_{i}}=\frac{1}{2} \frac{\partial \phi}{\partial x_{i}}
$$

$$
0 \geqq I_{2}+\frac{1}{2} \int_{Q} A_{\alpha \beta}^{i} u_{\alpha} u_{\beta} \frac{\partial \phi}{\partial x_{i}} d x d t .
$$

Combining (3.5) and (3.3) with (3.2) gives

$$
\begin{aligned}
\int_{Q} f_{\alpha} u_{\alpha} \phi d x d t \geqq & \left.\frac{1}{2} \int_{\Omega} E_{\alpha \beta} u_{\alpha} u_{\beta} \phi\right]_{0}^{T} d x \\
& -\frac{1}{2} \int_{Q}\left(E_{\alpha \beta} u_{\alpha} u_{\beta} \frac{\partial \phi}{\partial t}-A_{\alpha \beta}^{i} u_{\alpha} u_{\beta} \frac{\partial \phi}{\partial x_{i}}\right) d x d t
\end{aligned}
$$


But

$$
\begin{gathered}
\frac{\partial \phi}{\partial t}=\psi_{j}^{\prime}(\tau) c, \\
\frac{\partial \phi}{\partial x_{i}}=\psi_{\delta}^{\prime}(\tau)\left(x_{i}-x_{i}^{0}\right) /\left|x-x^{0}\right|=\psi_{\delta}^{\prime}(\tau) n_{i} \text { where } n_{i} n_{i}=1 .
\end{gathered}
$$

Hence, the integrand in the last integral is

$$
\psi_{\delta}^{\prime}(\tau)\left(E_{\alpha \beta} u_{\alpha} u_{\beta} c-A_{\alpha \beta}^{i} u_{\alpha} u_{\beta} n_{i}\right) \leqq 0,
$$

since $\psi_{\delta}^{\prime}(\tau) \leqq 0$ and $E_{\alpha \beta}(x) \xi_{\alpha} \xi_{\beta} c-A_{\alpha \beta}^{i}(x) \xi_{\alpha} \xi_{\beta} n_{i} \geqq 0$ for all $x \in \Omega, \xi \in R^{m}$ and $n_{i}$ with $n_{i} n_{i}=1$, by the definition of $c$. Combining (3.6) and (3.7) gives the estimate

$$
\begin{aligned}
\int_{Q} f_{\alpha} u_{\alpha} \phi d x d t \geqq & \frac{1}{2} \int_{\Omega} E_{\alpha \beta} u_{\alpha}(T) u_{\beta}(T) \phi(T) d x \\
& -\frac{1}{2} \int_{\Omega} E_{\alpha \beta} u_{\alpha}(0) u_{\beta}(0) \phi(0) d x .
\end{aligned}
$$

Finally, when $\delta \rightarrow 0, \phi$ tends boundedly to the characteristic function for $C\left(x^{0}, a\right)$. Hence, passage to the limit in (3.8) gives (3.1) with $k=0$, which completes the proof for the case $B=\frac{1}{2} \partial A^{i} / \partial x_{i}$. In the general case, a simple variant of this argument gives (3.1) with a nonzero $k$ (cf. [5, Theorem 6.1]).

\section{REFERENCES}

1. K. O. Friedrichs, Symmetric hyperbolic linear differential equations, Comm. Pure Appl. Math. 7 (1954), 345-393.

2. R. S. Phillips, Dissipative operators and hyperbolic systems of partial differential equations, Trans. Amer. Math. Soc. 90 (1959), 193-254.

3. C. H. Wilcox, The mathematical foundations of diffraction theory, Electromagnctic waves, pp. 65-97, Univ. of Wisconsin Press, Madison, Wis., 1962.

4. - Initial-boundary value problems for linear hyperbolic partial differential equations of the second order, Arch. Rational Mech. Anal. 10 (1962), 361-400.

5. - The domain of dependence inequality and initial-boundary value problems for symmetric hyperbolic systems, Technical Summary Rep. No. 333, Mathematics Research Center, U. S. Army, University of Wisconsin, Madison, Wis., August, 1962.

UNIVERSITY OF WISCONSIN 